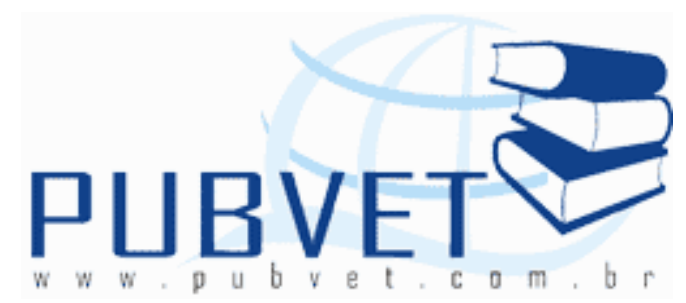

PUBVET, Publicações em Medicina Veterinária e Zootecnia.

Estudo da sutura do parênquima renal com fio absorvível sintético Aspectos histológicos

Paulo Cesar Silva1, Nelson Alfred Smith², Alberto Schanaider³, Manoel Luiz Ferreira ${ }^{4}$, Carlos Henrique Campello ${ }^{5}$, Fernando Breno Oliveira Ribeiro ${ }^{6}$

${ }^{1}$ Médico Veterinário, Professor Adjunto, Departamento de Cirurgia, Faculdade de Medicina, Universidade Federal do Rio de Janeiro.

2 Médico, Professor Associado, Departamento de Cirurgia, Faculdade de Medicina, Universidade Federal do Rio de Janeiro.

3 Médico, Professor Titular, Departamento de Cirurgia, Faculdade de Medicina, Universidade Federal do Rio de Janeiro.

4 Médico Veterinário, Professor Adjunto. Curso de Medicina Veterinária da Universidade Estadual de Santa Cruz (UESC).

5 Médico Veterinário, Pesquisador, Área de Anatomia PatológicaHistopatologia, CEPGM-Pesagro-Rio.

6 Discente Curso de Graduação, Programa de Iniciação Científica, Departamento de Cirurgia, Faculdade de Medicina, Universidade Federal do Rio de Janeiro.

\title{
Resumo
}

Os achados histopatológicos no parênquima renal quando da utilização de sutura com fio absorvível sintético não têm sido evidenciados. Sua observação possibilita o estudo da evolução cicatricial do parênquima, bem como a 
funcionalidade renal. O objetivo deste estudo é verificar quais as conseqüências para o parênquima renal quando da utilização de suturas com fio de absorvível sintético. No presente estudo foram utilizados 10 ratos machos com peso de $250 \mathrm{~g}$, origem SPF, distribuídos em 2 grupos $(n=5)$. Um com sutura utilizando poliglactina e outro controle. Foi realizada uma incisão no parênquima renal com $1 \mathrm{~cm}$ de extensão e $0,5 \mathrm{~cm}$ de profundidade, paralelo e afastado $0,5 \mathrm{~cm}$ do hilo, na face ventral. A sutura foi feita com pontos separados. O período de seguimento pós-operatório foi de 90 dias. Os dados analisados incluiram aderência, atrofia e resultados do exame histopatológico. A conclusão indica reação inflamatória nas áreas de fixação e ausência de resíduos do fio de sutura, mas com comprometimento glomerular.

Palavras-chave: sutura renal, fio absorvível sintético.

\title{
Study of renal parenchymal suturing wired absorbable synthetic
}

\section{Histological aspects}

\begin{abstract}
The interaction of synthetic absorbable suture with the renal parenchyma has not been demonstrated. This enables to check the aspect related to the influence on the healing and function of this organ. The aim of this study is to ascertain what consequences result when synthetic absorbable suture is applied in renal tissue. We used 10 male rats, weighing $250 \mathrm{~g}$, SPF origin, divided into 2 groups $(n=5)$. A sutured using polyglactin and other sham . The incision in the renal parenchyma than $1 \mathrm{~cm}$ long and $0.5 \mathrm{~cm}$ deep, $0.5 \mathrm{~cm}$ away and parallel to the hilum, on the ventral kidney. Followed by interrupted suture. The postoperative period of 90 days. The data analyzed include adhesion, atrophy and histopathological study. The finding indicates inflammatory reaction in the attachment areas and absence of residues of the suture, but with glomerular empairement.
\end{abstract}

Keywords: renal suture, absorbable synthetic suture. 


\section{Introdução}

A sutura aplicada ao tecido renal, após nefrotomia ou nefrectomia parcial, inclui elementos estruturais que podem comprometer a funcionalidade do órgão. Estão indicados os fios de sutura absorvíveis tanto naturais quanto sintéticos ${ }^{1}$. Os fios sintéticos foram lançados no início da década de 1970, tendo como composição polímeros de peso molecular elevado, lineares, com capacidade de liquefação e com moldagem filamentar de diâmetros variados, tendo como padrão o ácido glicólico. São implicados como tendo capacidade litogênica, quando do contato com urina. O processo de como isto ocorre não está esclarecido².

Na cicatrização, são caracterizados como tendo absorção rápida devido à hidrólise, ocasionando mínima reação inflamatória ${ }^{3}$. Cabe ressaltar que não se tem informações sobre o destino dos fragmentos do fio utilizado em órgãos emunctórios, como os rins.

O objetivo do presente estudo é analisar a interação entre o fio de sutura absorvível sintético e o parênquima renal.

\section{Método}

Projeto aprovado pela Comissão de Ètica- Cepal, com o número 13/10. Ratos oriundos do biotério do Centro de Cirurgia Experimental, da Faculdade de Medicina da UFRJ. Mantidos no referido biotério pelo período do experimento, em condições ambientais estáveis, ração industrial e água ad libidum. Experimento realizado sob controle veterinário. Foram utilizados dez ratos, machos, com peso médio de 250 gramas, linhagem Wistar, origem SPF. Separados em dois grupos de cinco animais, sendo um grupo experimental $(n=5)$ e outro grupo simulado $(n=5)$.

Anestesia geral com a associação de quetamina, $10 \mathrm{mg} / 100 \mathrm{~g}$ de peso e xilazina, $1 \mathrm{mg} / 100 \mathrm{~g}$ de peso, via intraperitoneal. 
Os animais foram submetidos à laparotomia mediana com acesso ao rim esquerdo. No grupo simulado foi realizada somente dissecção do tecido perirrenal e isolamento do rim. No grupo experimental, após isolamento do rim é realizada uma incisão com $1 \mathrm{~cm}$ de extensão e $0,5 \mathrm{~cm}$ de profundidade, paralelo ao hilo renal e afastado do mesmo, na face ventral do rim. A localização da incisão foi planejada para minimizar a possibilidade de envolvimento dos vasos interlobulares e assegurar maior proximidade com o sistema coletor. A sutura foi efetuada com fio de poliglactina 910, constituída de 5 pontos separados no parênquima. A laparorrafia foi realizada por planos, compreendendo linha média do plano músculo-aponeurótico, subcutâneo e pele.

No pós-operatório utilizou-se analgesia por 5 dias com dipirona gotas, diluída na água de beber. $\mathrm{O}$ acompanhamento pós-operatório foi de 90 dias. A indução da morte foi realizada com tiopental sódico a 2,5\%, aplicado por via intraperitoneal.

Os dados a serem coletados incluiram as alterações renais e peri-renais, como aderência e atrofia. Os órgãos retirados para exame histopatológico foram fixados em solução de formalina à $10 \%$ tamponada com salina. Em seguida foram incluídos em blocos de parafina, corados pela técnica de hematoxilina-eosina e examinados em microscópico óptico.

\section{Resultados}

Nos cinco animais do grupo simulado, não foram observadas alterações renais. Nos animais do grupo experimental, com sutura absorvível, foram observadas aderência e atrofia nas áreas da sutura. (Fig.1 e Fig.2)

No exame histopatológico utilizou-se coloração pela hematoxilina-eosina $(\mathrm{HE})$, e análise sob microscopia óptica. Na área de contato do fio de sutura com o parênquima renal observou-se a presença de fibrose (Fig 3). 
SILVA, P.C. et al. Estudo da sutura do parênquima renal com fio absorvível sintético Aspectos histológicos. PUBVET, Londrina, V. 7, N. 21, Ed. 244, Art. 1613, Novembro, 2013.

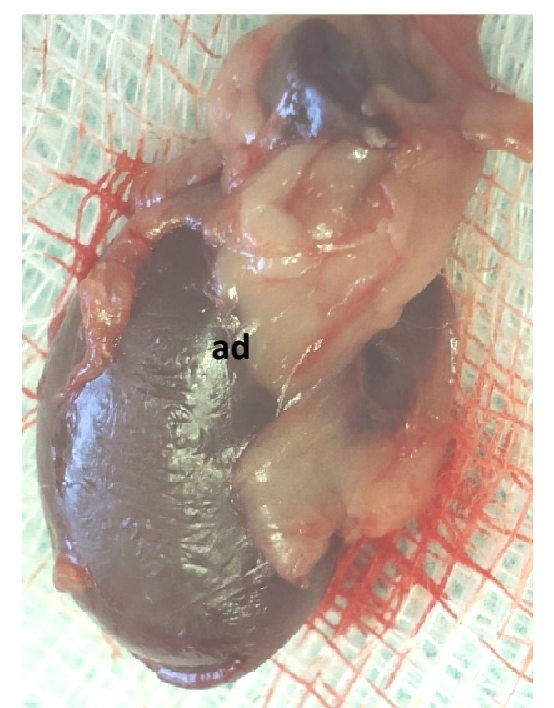

Figura 1. Rim. Aderência (ad)

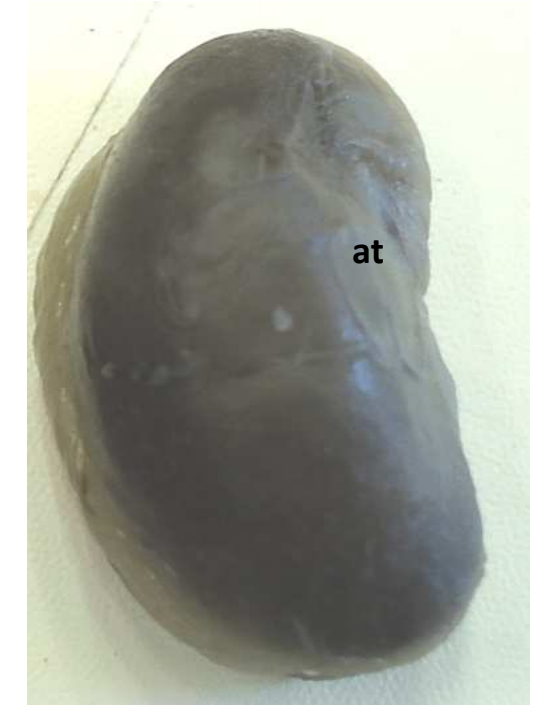

Figura 2. Rim. Atrofia (at)

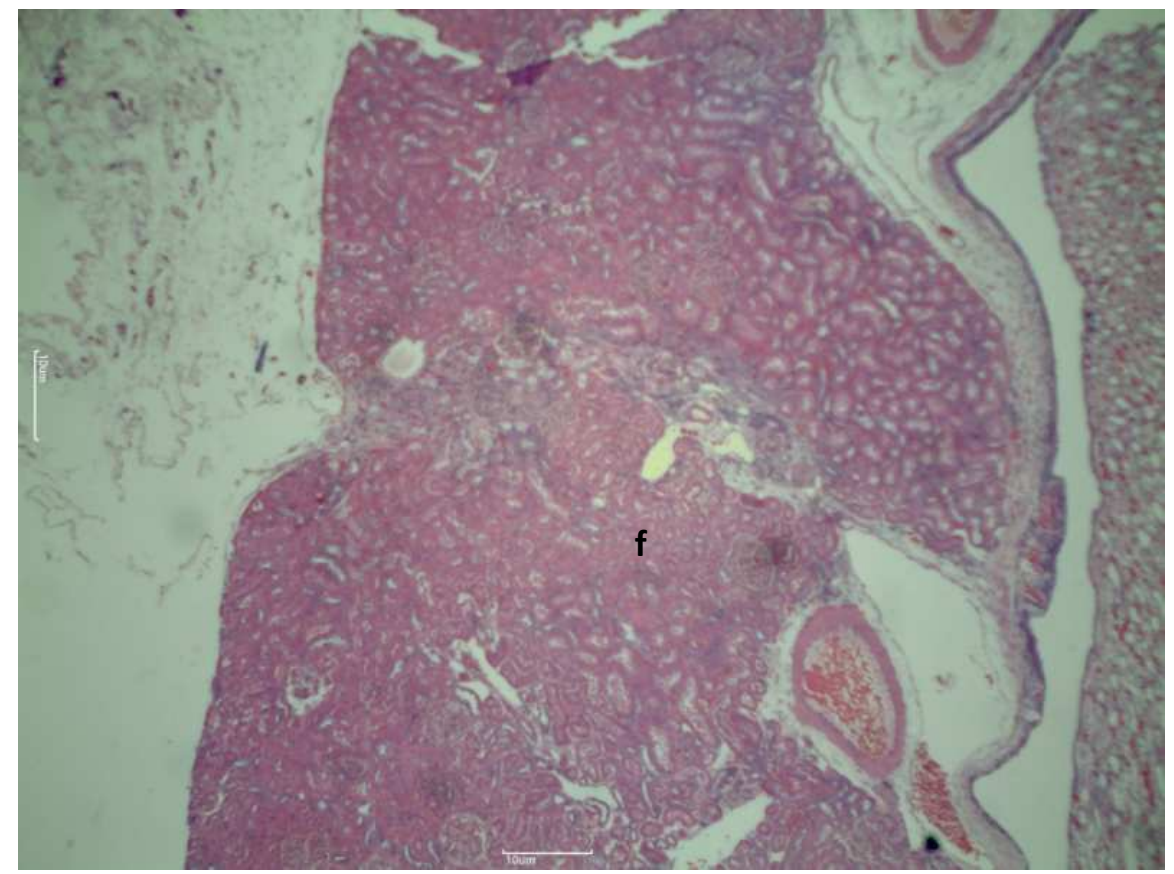

Figura 3-fotomicrografia, HE, X10-. Área de contato do fio com o parênquima evidenciando fibroplasia no parênquima renal $(F)$. 
A presença de fibrose foi caracterizada pela proliferação de fibroblastos no interstício, envolvendo alguns túbulos renais e glomérulos. Observou-se atrofia glomerular, junto às áreas da sutura. No parênquima renal envolvido pela sutura não foram identificados resíduos dos fios. As demais áreas do tecido renal estavam preservadas. Fig. 4

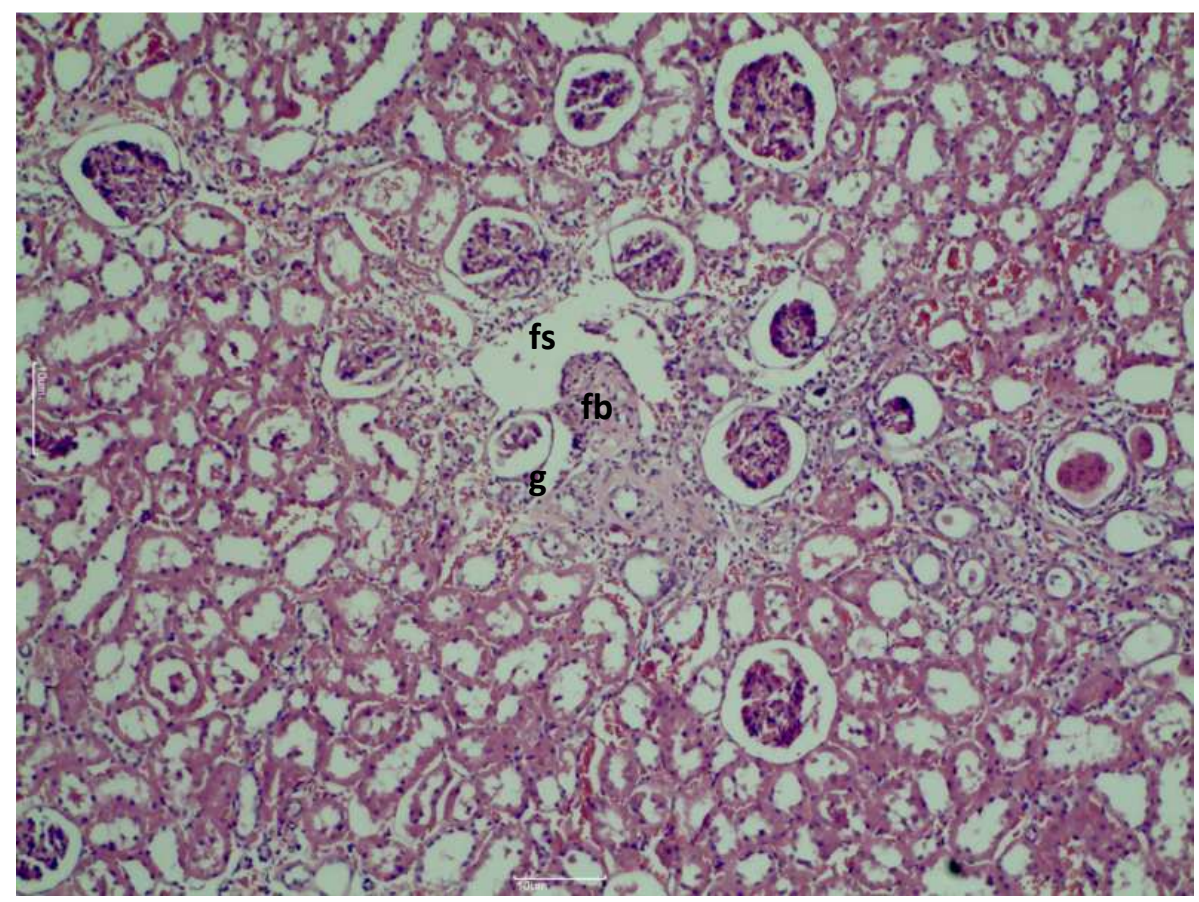

Figura 4- fotomicrografia, HE, X40-Parênqima renal, com preseça de fibroblasto(fb) envolvendo túbulos renais $(t)$ e glomérulos atrofiados $(\mathrm{g})$. Área de localização do fio de sutura(fs).

\section{Discussão}

Em função das características anatômicas do rim, as suturas aplicadas ao parênquima renal são realizadas de tal forma a evitar lesões vasculares e ao sistema coletor, principalmente quanto ao nível de aplicação desta sutura, indo da cortical até a medular ${ }^{4}$. Estudos sobre sutura renal utilizando fios de sutura de poliglactina, estão voltados para sua aplicação na nefrectomia parcial vídeolaparoscópica, em relação ao controle do tempo de isquemia e sangramento 
durante o procedimento operatório, mas não descrevem as alterações ocasionadas no parênquima renal ${ }^{5}$. As alterações microscópicas quando do uso de fio de poliglactina, como infiltração de células mononucleadas e fibrose intersticial, observadas no presente estudo, condizem com os dados obtidos por outros autores, em pesquisas sobre reconstrução renal e em modelos de insuficiência renal ${ }^{6,7}$.

As alterações renais, como a fibrose e atrofia glomerular, ocasionadas pela passagem e permanência do fio na fase de absorção, perduram mesmo após a ausência do fio. Isto é indicativo de que o processo cicatricial é independente da constituição do fio de poliglactina.

Outro aspecto relevante ligado ao tipo de fio de sutura e que não tem sido levantado nas pesquisas, não está relacionado só com a presença do fio no tecido renal, mas também com o processo de absorção e destino dos seus componentes. Isto tem interesse devido ao fato de que os fios absorvíveis sintéticos, em contato com a urina possuem capacidade litogênica ${ }^{8}$. O fato de não se constatar fragmentos dos fios após 90 dias, permite questionamentos sobre o destino deste material.

Nas cirurgias renais, considerando fios de sutura retidos (não absorvidos), os fios de poliglactina são o segundo em frequência e atuam como núcleo para formação de cálculos, de acordo com uma revisão da literatura sobre corpo estranho renal de etiologia iatrogênica?

Da mesma forma, alguns autores mostram que fios de sutura aplicados ao parênquima renal e não absorvidos podem formar granulomas que mimetizam massas renais ${ }^{10}$.

\section{Conclusão}

O fio de poliglactina 910 promove fibrose, com comprometimento glomerular local e sem presença de resíduo, quando utilizado na sutura do parênquima renal. 


\section{Referências Bibliográficas}

1 - Ribeiro, F B O et al.(2012). Sutura com fios absorvíveis no parênquima renal no rato. In: XXXIV Jornada Giulio Massarani de Iniciação Científica, Artística e Cultural - Centro de Ciências da Saúde - Universidade Federal do Rio de Janeiro. Rio de Janeiro. Livro de Resumos.

2 - Hering, FLO, Gabor,S, Rosenberg,D (1990). Estudo in vivo no rato da litogenesia dos fios de poligliconato, categute cromado e poliglactina 910 em urina estéril e em urina infectada por E.coli e P. mirabilis. Acta Cirurgica Brasileira. 5:105-108 p.

3 - Bezwada, R S et al. (1995). Monocryl suture, a new ultra-pliable absorbable monofilament suture. Biomoterials 16:1141-1148 p.

4 - L'esperance, JO et al. (2010). Do nonspecific deep corticomedullary sutures performed during partial nephrectomy adequately control major vascular and collecting system injury. British Journal of Urology International. Feb;105 (3): 411-5 p.

5 - Kawa G, et al. ( 2010). Uninterrupted suturing of renal parenchyma in laparoscopic partial nephrectomy decreases renal ischemic time and intraoperative blood loss. International Journal of Urology. Apr; 17(4):382-4p.

6 - Akay AF, et al. (2005). Reconstruction techniques in renal injury. Saudi Medical Journal. Nov;26 (11):1750-4p.

7 - Costa, A F N, et al.(2009). Modelo cirúrgico de insuficiência renal crônica. Estudo em coelhos. Revista do Colégio Brasileiro de Cirurgiões. 36 (1). Rio de Janeiro.

8 - Conilho, L C et al. (2011). Comparação de técnica de sutura com fios absorvíveis na parede vesical. Estudo experimental no rato. In:XXXIII Jornada Giulio Massarani de Iniciação Científica, Artística e Cultural - Centro de Ciências da Saúde - Universidade Federal do Rio de Janeiro. Rio de Janeiro. Livro de Resumos, p.358.

9 - D.B. Hennessey, A.Z. Thomas, T.H. Lynch, R.G. Case (2012). Retained Upper Genitourinary Gossypiboma Can Mimic Renal Neoplasms. A Review Of The Literature. The Internet Journal of Urology. 9 (2).

10 - Dogra PN, Tandon S, Ansari MS, Anupama, Chopra P. (2005). Suture foreign body granuloma masquerading as renal neoplasm. Int Urol Nephrol. 37(1):27-9. 